\title{
Serum HOTAIR as a novel diagnostic biomarker for esophageal squamous cell carcinoma
}

\author{
Wenjian Wang ${ }^{\dagger}$, Xiaotian $\mathrm{He}^{\dagger}$, Zehua Zheng, Xiaofan Ma, Xueting Hu, Duoguang Wu ${ }^{*}$ and Minghui Wang ${ }^{*}$
}

\begin{abstract}
Background: Early diagnosis of esophageal squamous cell carcinoma (ESCC) is an important issue to improve the prognosis. HOX transcript antisense RNA (HOTAIR), a long noncoding RNA (IncRNA) expressed from the HOXC locus, has been recently revealed as an oncogenic regulator in ESCC. This study aimed to investigate whether serum HOTAIR is involved in the diagnosis of ESCC.

Methods: In this study, we detected serum HOTAIR expression in 50 patients with ESCC (including 42 tumor resection and 8 without surgery) and 20 healthy volunteers to investigate the role of serum HOTAIR in ESCC using the quantitative real-time polymerase chain reaction (qRT-PCR) method.

Results: Clinical data indicated that serum HOTAIR were correlated with TNM stage. The expression level of serum HOTAIR $(0.189 \pm 0.010)$ was significantly higher in ESCC patients compared with that of healthy controls $(0.055 \pm 0.008, P<0.01)$. The ROC curve analysis yielded an area under the ROC curve (AUC) value of 0.793 (95\% Cl: 0 . 692 to $0.895, P<0.01$ ). Also, the serum HOTAIR expression level decreased obviously in postoperative samples (one month after the surgery) compared to preoperative specimens. Moreover, there was a significant correlation between serum HOTAIR expression and the expression of HOTAIR in ESCC tissue according to Pearson correlation analysis.
\end{abstract}

Conclusions: Our study, for the first time, demonstrated that serum HOTAIR might serve as a potential biomarker for the diagnosis of ESCC.

Keywords: Esophageal squamous cell carcinoma, Long noncoding RNA, HOTAIR, Biomarker, Diagnosis

\section{Introduction}

Esophageal squamous cell carcinoma (ESCC) is the major histopathological subtype of esophageal cancer [1,2]. Due to its tumor aggressiveness and therapeutic difficulties, ESCC ranks eighth in incidence and sixth in cancer death worldwide [3]. Approximately, 300,000 people die from esophageal cancer in the world annually [4]. Although the advances in medical and surgical treatments, most ESCC patients are diagnosed at an advanced stage with a poor prognosis, an overall five-year survival ranging from $15-25 \%$ [5]. Therefore, early detection of primary tumors provides effective treatments and timely interventions,

\footnotetext{
*Correspondence: b_x_s0714@sina.com; j_c_r0714@sina.com

'Equal contributors

Guangdong Provincial Key Laboratory of Malignant Tumor Epigenetics and Gene Regulation, Department of Thoracic surgery, Sun yat-sen memorial hospital, Sun yat-sen University, NO.107 of Yanjiangxi Road, Guangzhou 510120, China
}

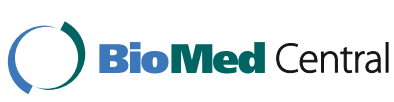

(c) The Author(s). 2017 Open Access This article is distributed under the terms of the Creative Commons Attribution 4.0 International License (http://creativecommons.org/licenses/by/4.0/), which permits unrestricted use, distribution, and reproduction in any medium, provided you give appropriate credit to the original author(s) and the source, provide a link to the Creative Commons license, and indicate if changes were made. The Creative Commons Public Domain Dedication waiver (http://creativecommons.org/publicdomain/zero/1.0/) applies to the data made available in this article, unless otherwise stated. gen (SCC), and p53, which are now clinically used as ESCC tumor markers, are inadequate for identifying subclinical patients with early tumors and predicting disease recurrence [6].

Noncoding RNAs (ncRNAs), such as microRNAs, small interfering RNAs, and long noncoding RNAs (lncRNAs), play important regulatory roles in the development of many diseases especially in cancers $[7,8]$. The lncRNAs, defined as RNA molecules $>200$ nucleotides in length, have been shown to be involved in the regulation of gene expression in both tumor-suppressive and oncogenic pathways at epigenetic level, transcriptional level, and posttranscriptional level $[9,10]$. Mounting evidence supported the potential markers of lnvRNAs in tumor diagnosis and prognosis. For instance, increased expression 
of BC200RNA has recently been proposed as a potential novel molecular marker for breast cancer [11, 12], while MALAT-1 gene overexpression suggests poor prognosis in lung cancer patients [13]. However, lncRNA expressions in blood have not been investigated as potential novel biomarkers for ESCC diagnosis or prognosis.

HOX antisense intergenic RNA (HOTAIR), a lncRNA localized on chromosome within the homeobox $\mathrm{C}$ (HOXC) gene cluster [14] was initially discovered as a repressor of the HOXD genes [15]. Recently, researchers found that HOTAIR was significantly overexpressed in a variety of tumors and was able to induce the proliferation and metastasis of these tumors [16]. Our previous study [17] found that high expression levels of HOTAIR correlated clinically with ESCC progression. Moreover, HOTAIR contributes to the malignant phenotype of ESCC cells through its regulation of diverse cellular processes, including migration, invasion, and proliferation [17]. So, this study aimed to investigate the expression of serum HOTAIR in ESCC patients and to evaluate its diagnostic value in the early diagnosis of ESCC.

\section{Methods}

\section{Patients and blood samples}

A total of 50 consecutive patients with ESCC were recruited at the Sun Yat-Sen Memorial Hospital of Sun Yat-Sen University from June 2013 to January 2016. All of these included subjects were newly diagnosed and previously untreated (including surgery, chemotherapy, and radiotherapy). The data including age, gender, histologic grade, $\mathrm{T}$ stage, lymph node metastasis, and TNM stage were recorded. Tumors were staged according to the TNM staging system of the American Joint Committee on Cancer (AJCC) [18]. As a control, 20 individuals who sought a routine health check-up at the same period and did not have any esophageal diseases or other cancerous diseases were recruited. All blood samples were collected with informed consent and agreement, according to protocols approved by the Ethics Committee of the Sun Yat-Sen Memorial Hospital of Sun Yat-Sen University.

Of those 50 patients with ESCC, 42 underwent surgery, and 8 did not undergo surgery because of distant metastasis. Postoperative venous blood samples from 42 patients were collected 1 month after the surgery. Preoperative venous blood samples of 50 ESCC patients and 20 healthy controls were also collected. Blood samples were centrifuged at $2000 \mathrm{r} / \mathrm{min}$ for $10 \mathrm{mins}$ at $4{ }^{\circ} \mathrm{C}$, and the serum was collected and stored at $-80{ }^{\circ} \mathrm{C}$ until further processing. Of the 42 patients who underwent surgery, 20 ESCC tissues were collected during the operation. Fresh tissue samples were frozen within 30 min after surgery and stored in liquid nitrogen.
Tissue sections from each ESCC sample were reviewed and classified by a pathologist.

RNA extraction, reverse transcription, and quantitative real-time PCR(qRT-PCR)

Total RNA was extracted from each serum and ESCC tissue sample using Trizol LS reagent (Invitrogen life technologies, USA) and then reverse-transcribed with a SuperScriptTM III Reverse Transcriptase (Invitrogen) according to the manufacturer's instructions. After that, qRT-PCR was performed to quantify the expression levels of lncRNAs using Gene Amp PCR System 9700 (Applied Biosystems, CA, USA) and the 2X PCR Master mix (KANGCHENG, Shanghai, China). The HOTAIR qPCR primers used are forward,5'-GGAAAGATCCAA ATGGGACCA-3'; reverse,5'CTAGGAATCAGCACGA AGCAAA-3'. U6 was evaluated as a housekeeping gene for the qPCR reactions. The U6 qPCR primers used are 5'-GCTTCGGCAGCACATATACTAAAAT-3'; reverse,5'CGCTTCACGAATTTGCGTGTCAT-3'. The expression level of HOTAIR in each sample was normalized to that of the internal control U6. HOTAIR absolute expression levels were calculated by the the $2-\Delta \Delta \mathrm{Ct}$ method.

\section{Statistical analysis}

SPSS Statistics software version 17.0 was used for the statistical analysis. All data was analyzed by normal distribution test. Mean \pm standard deviation (SD) was used to represent measurement data with normal distribution. Comparison of measurement data of normal distribution was analyzed by student's $t$-test. Pearson correlation analysis was used to assess the association between two continues variables. Receiver operating characteristics (ROC) curve was plotted to determine how well the expression level of serum HOTAIR discriminated between tumor samples and healthy control samples. An AUC-ROC value of $>0.7$ was taken to indicate reasonable biomarker performance. ROC curves optimal cut-off values were defined as the point that maximized the Youden index, defined as (sensitivity + specificity)-1. A $P$-value of $<0.05$ was considered statistically significant.

\section{Results \\ Clinicopathological characteristics and serum HOTAIR expression levels of ESCC patients}

As shown in Table 1, the expression level of serum HOTAIR was closely associated with TNM stage $(P=$ 0.002). Furthermore, patients with distant metastasis showed significantly higher HOTAIR expression level than patients without distant metastasis $(0.248 \pm 0.028$ versus $0.189 \pm 0.010, P=0.004)$. However, there was no correlation detected in the expression level of serum 
Table 1 Clinicopathological characteristics and serum HOTAIR expression levels of ESCC patients

\begin{tabular}{|c|c|c|c|}
\hline Variables & No. of patients & $\begin{array}{l}\text { Serum HOTAIR expression } \\
\text { (mean } \pm \text { SD) }\end{array}$ & $P$ value \\
\hline Age, year & & & 0.739 \\
\hline$\geq 60$ & 23 & $0.192 \pm 0.014$ & \\
\hline$<60$ & 19 & $0.185 \pm 0.015$ & \\
\hline Gender & & & 0.820 \\
\hline Male & 33 & $0.190 \pm 0.013$ & \\
\hline Female & 9 & $0.184 \pm 0.013$ & \\
\hline TNM stage & & & 0.002 \\
\hline । & 6 & $0.134 \pm 0.050$ & \\
\hline$\|$ & 10 & $0.181 \pm 0.051$ & \\
\hline III & 26 & $0.204 \pm 0.069$ & \\
\hline IV & 8 & $0.261 \pm 0.022$ & \\
\hline T stage & & & 0.140 \\
\hline $\mathrm{T} 1-\mathrm{T} 2$ & 11 & $0.144 \pm 0.075$ & \\
\hline T3 & 31 & $0.174 \pm 0.083$ & \\
\hline N stage & & & 0.053 \\
\hline NO & 15 & $0.160 \pm 0.055$ & \\
\hline N1 & 20 & $0.181 \pm 0.061$ & \\
\hline N2-N3 & 7 & $0.224 \pm 0.037$ & \\
\hline M stage & & & 0.004 \\
\hline MO & 42 & $0.189 \pm 0.010$ & \\
\hline M1 & 8 & $0.248 \pm 0.028$ & \\
\hline Differentiation & & & 0.871 \\
\hline Well & 12 & $0.197 \pm 0.070$ & \\
\hline Moderate & 19 & $0.185 \pm 0.061$ & \\
\hline Poor & 11 & $0.186 \pm 0.061$ & \\
\hline
\end{tabular}

HOTAIR with age, gender, lymph node metastasis, depth of invasion and degree of tumor differentiation.

The serum HOTAIR expression level of patients was positively correlated with the expression of HOTAIR in ESCC tissue

HOTAIR expression levels in both ESCC tissues and serum of ESCC patients were analyzed to detect their relationship. As shown in Fig. 1a, we found that ESCC tissue had a significantly increased HOTAIR expression compared with the level in serum $(P<0.01)$. Also, serum HOTAIR expression level was positively associated with the expression of HOTAIR in ESCC tissue $(r=0.6904$, $P<0.01)$. It suggested that serum HOTAIR might serve as a promising biomarker.

\section{Upregulation of serum HOTAIR level in ESCC patients}

The expression level of serum HOTAIR in 50 ESCC patients and 20 healthy controls were detected to determine whether serum HOTAIR expression levels are higher in patients with ESCC. As shown in Fig. 1b, the serum of ESCC had a significantly increased HOTAIR expression compared with the level of healthy controls $(0.189 \pm 0.010$ versus $0.055 \pm 0.008, P<0.01)$.

\section{ROC curve of serum HOTAIR level in the diagnosis of ESCC}

We further analyzed ROC curve of serum HOTAIR level to assess its diagnostic value (Fig. 2a). We found that serum HOTAIR level could differentiate ESCC patients from healthy controls, with an AUC of 0.793 (95\% CI: 0.692 to $0.895, P<0.01)$. And the optimal cut-off values were 0.094 (sensitivity $56.0 \%$, specificity $90.0 \%$ )

\section{The correlation of serum HOTAIR expression level} between preoperative and postoperative patients in ESCC The postoperative venous blood samples from 20 patients were collected 1 month after surgery. The expression level of serum HOTAIR in postoperative samples decreased significantly compared with the level of preoperative specimens $(0.083 \pm 0.008$ versus $0.189 \pm 0.010, P<0.01)$ (Fig. $2 \mathrm{~b}$ and $\mathrm{c}$ ).

\section{Discussion}

ESCC is an aggressive tumor with rapid growth rate and high opportunity of regional and distant metastasis, which contributes to the poor prognosis. Therefore, it is an urgent need to find an effective biomarker for ESCC so that the dismal tumor can be treated at the early stage. Circulating lncRNAs has been confirmed that they can be remarkably stable in serum and be detected in human peripheral blood in spite of the high amounts of RNase in the blood of cancer patients [19]. Clinically, overexpression of HOTAIR is a powerful predictor of overall survival and progression for several cancers including gastrointestinal stromal tumors [20], breast cancer [21], squamous cell carcinoma [22], nasopharyngeal carcinoma laryngeal [23], colon cancer [24], hepatocellular carcinoma [25, 26] and pancreatic cancer [27]. In our previous study, HOTAIR, a lncRNA has been considered as a prognostic marker for ESCC [17]. Here we found that the serum HOTAIR also showed great promise as a novel diagnostic biomarker of ESCC.

We measured the expression of HOTAIR in ESCC tissue and serum of 50 ESCC patients. The results revealed that ESCC tissue had a significantly increased HOTAIR expression compared with that of serum. Also, serum HOTAIR expression level was positively associated with the expression of HOTAIR in ESCC tissue. Combined with our previous study, it suggested that serum HOTAIR could serve as a promising biomarker. The qRT-PCR method was performed to examine the expression level of serum HOTAIR in 50 ESCC patients and 20 healthy controls to determine whether serum HOTAIR expression levels are higher in 

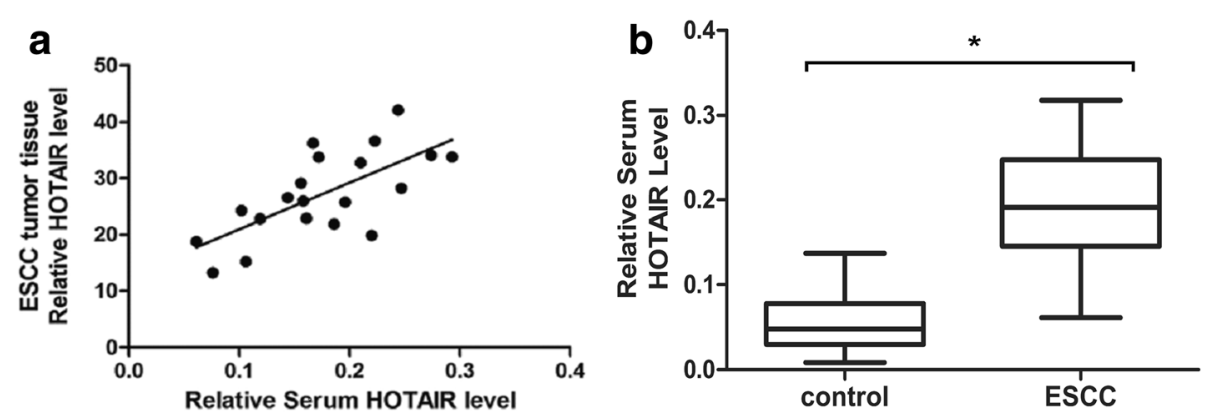

Fig. 1 HOTAIR expression levels from different sources and patients. a Pearson correlation analysis of HOTAIR expression levels in ESCC tissue and serum; b Comparison of serum HOTAIR expression level between ESCC patients and healthy controls

patients with ESCC, The results showed that serum of ESCC had a significantly increased HOTAIR expression compared with that of healthy controls. Moreover, we found that serum HOTAIR was significantly correlated with $\mathrm{M}$ stage and TNM stage. All these evidence further confirmed an important role of HOTAIR on the ESCC progression. ROC curve analysis showed serum HOTAIR could differentiate ESCC patients from healthy controls, with an AUC of 0.793. It revealed that serum HOTAIR could be used as a promising diagnostic indicator for patients with ESCC.

The release of nucleic acids into the blood is thought to be related to necrosis tumor cells from the tumor microenvironments [28]. Our results showed that the expression level of serum HOTAIR in postoperative samples decreased significantly compared with that of preoperative specimens. It revealed that the origin of HOTAIR derived from tumor cells and serum HOTAIR was down-regulated after the tumor resection. Therefore, serum HOTAIR expression level can serve as the indicator for cancer recurrence of ESCC patients who undergo tumor resection.

However, certain limitations in our study should be addressed as follows. The population of enrolled patients and controls was relatively small, which might result in bias in the final results. Besides, it is a pity that we could not gain enough data to perform survival analysis. Therefore, a longer follow-up study is needed to assess the prognostic significance of serum HOTAIR in ESCC.

\section{Conclusion}

For the first time, the results of our study indicated that the expression of HOTAIR in serum could be used as a novel diagnostic biomarker for ESCC.
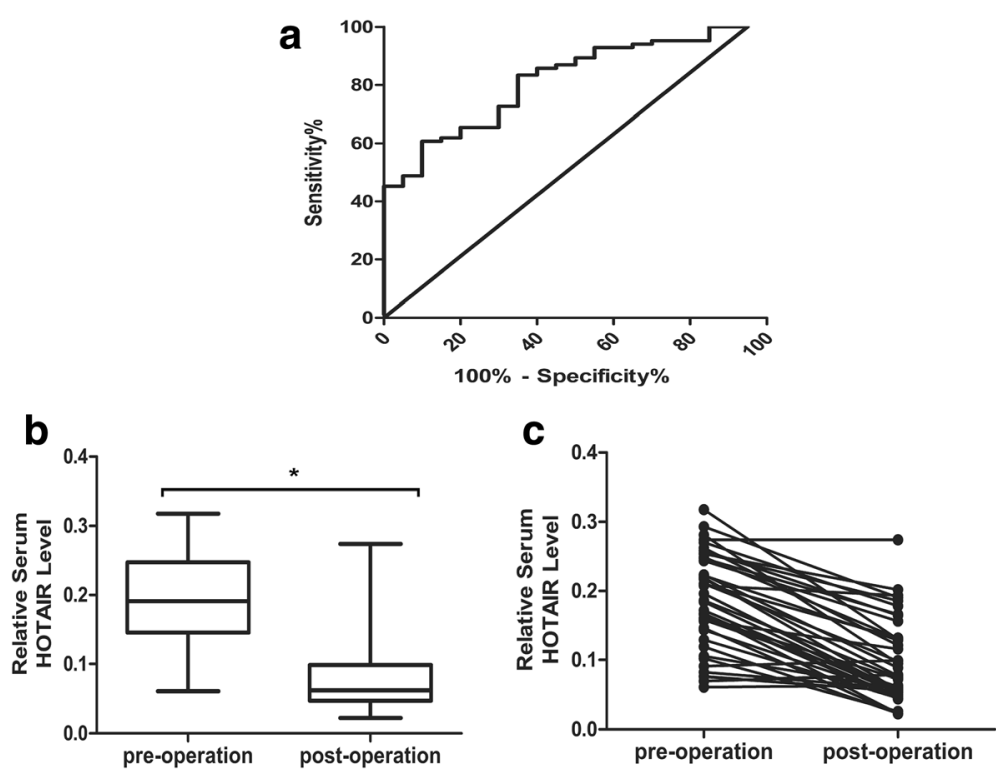

Fig. 2 Serum HOTAIR levels as a diagnostic biomarker. a ROC curve analysis for serum HOTAIR in the diagnosis of ESCC; (b, c) Comparison of serum HOTAIR expression levels between preoperative and postoperative samples 


\section{Acknowledgements}

Not applicable.

\section{Funding}

This work was supported by The National Natural Science Foundation of China. (No. 81372567); Grant [2013] 163 from Key Laboratory of Malignant Tumor Molecular Mechanism and Translational Medicine of Guangzhou Bureau of Science and information Technology; Grant KLB09001 from the key Laboratory of Malignant Tumor Gene Regulation and Target Therapy of Guangdong Higher Education institutes.

\section{Availability of data and materials}

Not applicable.

\section{Authors' contributions}

MW, DW: guarantor of integrity of the entire study; MW: study concepts; $\mathrm{MW}$ : study design; $\mathrm{WW}, \mathrm{XH}$ : definition of intellectual content; $\mathrm{WW}, \mathrm{XH}$ : literature research; $W W, X H, Z Z, X M, X H, D W, M W$ : clinical studies; $W W, X H$, ZZ, XM, XH, DW, MW: experimental studies; XH: data acquisition; WW: data analysis; WW: statistical analysis; ZZ, XM: manuscript preparation; XH: manuscript editing; MW, DW: manuscript review. All authors read and approved the final manuscript.

\section{Authors' information}

Please see the title page.

\section{Competing interests}

The authors declare that they have no competing interest.

\section{Consent for publication}

Not applicable.

\section{Ethical approval and consent to participate}

Not applicable.

\section{Publisher's Note}

Springer Nature remains neutral with regard to jurisdictional claims in published maps and institutional affiliations.

\section{Received: 2 November 2016 Accepted: 27 March 2017}

Published online: 04 April 2017

\section{References}

1. Matsushima $\mathrm{K}$, Isomoto $\mathrm{H}$, Yamaguchi $\mathrm{N}$, Inoue N, Machida $\mathrm{H}$, Nakayama T, Hayashi T, Kunizaki M, Hidaka S, Nagayasu T, et al. MiRNA-205 modulates cellular invasion and migration via regulating zinc finger E-box binding homeobox 2 expression in esophageal squamous cell carcinoma cells. J Transl Med. 2011:9:30.

2. Nagaraja V, Eslick GD. Forthcoming prognostic markers for esophageal cancer: a systematic review and meta-analysis. J Gastrointest Oncol. 2014;5: 67-76.

3. Rustgi A, El-Serag HB. Esophageal carcinoma. N Engl J Med. 2015;372:1472-3.

4. Chai J, Jamal MM. Esophageal malignancy: a growing concern. World J Gastroenterol. 2012;18:6521-6.

5. Pennathur A, Gibson MK, Jobe BA, Luketich JD. Oesophageal carcinoma. Lancet. 2013;381:400-12.

6. Shimada H, Takeda A, Arima M, Okazumi S, Matsubara H, Nabeya Y, Funami Y, Hayashi H, Gunji Y, Suzuki T, et al. Serum p53 antibody is a useful tumor marker in superficial esophageal squamous cell carcinoma. Cancer. 2000;89: 1677-83.

7. Malone CD, Hannon GJ. Small RNAs as guardians of the genome. Cell. 2009; 136:656-68

8. Moazed D. Small RNAs in transcriptional gene silencing and genome defence. Nature. 2009:457:413-20.

9. Wang KC, Chang HY. Molecular mechanisms of long noncoding RNAs. Mol Cell. 2011:43:904-14

10. Guttman M, Donaghey J, Carey BW, Garber M, Grenier JK, Munson G, Young $G$, Lucas $A B, A c h R$, Bruhn $L$, et al. lincRNAs act in the circuitry controlling pluripotency and differentiation. Nature. 2011;477:295-300.
11. Tsai MC, Manor O, Wan Y, Mosammaparast N, Wang JK, Lan F, Shi Y, Segal E, Chang HY. Long noncoding RNA as modular scaffold of histone modification complexes. Science. 2010;329:689-93.

12. lacoangeli A, Lin Y, Morley EJ, Muslimov IA, Bianchi R, Reilly J, Weedon J, Diallo R, Bocker W, Tiedge H. BC200 RNA in invasive and preinvasive breast cancer. Carcinogenesis. 2004;25:2125-33.

13. Ji P, Diederichs S, Wang W, Boing S, Metzger R, Schneider PM, Tidow N, Brandt B, Buerger $H$, Bulk E, et al. MALAT-1, a novel noncoding RNA, and thymosin beta4 predict metastasis and survival in early-stage non-small cell lung cancer. Oncogene. 2003;22:8031-41.

14. Bhan A, Hussain I, Ansari Kl, Kasiri S, Bashyal A, Mandal SS. Antisense transcript long noncoding RNA (IncRNA) HOTAIR is transcriptionally induced by estradiol. J Mol Biol. 2013;425:3707-22.

15. Rinn JL, Kertesz M, Wang JK, Squazzo SL, Xu X, Brugmann SA, Goodnough $\mathrm{LH}$, Helms JA, Farnham PJ, Segal E, Chang HY. Functional demarcation of active and silent chromatin domains in human HOX loci by noncoding RNAs. Cell. 2007:129:1311-23.

16. Tano K, Akimitsu N. Long non-coding RNAs in cancer progression. Front Genet. 2012;3:219

17. Lv XB, Lian GY, Wang HR, Song E, Yao H, Wang MH. Long noncoding RNA HOTAIR is a prognostic marker for esophageal squamous cell carcinoma progression and survival. PLoS One. 2013;8, e63516.

18. Edge SB, Compton CC. The American Joint Committee on Cancer: the 7th Edition of the AJCC Cancer Staging Manual and the Future of TNM. Ann Surg Oncol. 2010;17:1471-4.

19. Tong YS, Wang XW, Zhou XL, Liu ZH, Yang TX, Shi WH, Xie HW, LV J, Wu QQ, Cao XF. Identification of the long non-coding RNA POU3F3 in plasma as a novel biomarker for diagnosis of esophageal squamous cell carcinoma. Mol Cancer. 2015;14:3

20. Niinuma T, Suzuki H, Nojima M, Nosho K, Yamamoto $H$, Takamaru $H$, Yamamoto E, Maruyama R, Nobuoka T, Miyazaki Y, et al. Upregulation of miR-196a and HOTAIR drive malignant character in gastrointestinal stromal tumors. Cancer Res. 2012:72:1126-36.

21. Lu L, Zhu G, Zhang C, Deng Q, Katsaros D, Mayne ST, Risch HA, Mu L, Canuto EM, Gregori G, et al. Association of large noncoding RNA HOTAIR expression and its downstream intergenic CpG island methylation with survival in breast cancer. Breast Cancer Res Treat. 2012;136:875-83.

22. Antonelli M, Sandroni C. Hydroxyethyl Starch for Intravenous Volume Replacement More Harm Than Benefit. JAMA. 2013;309:723-4.

23. Nie Y, Liu X, Qu S, Song E, Zou H, Gong C. Long non-coding RNA HOTAIR is an independent prognostic marker for nasopharyngeal carcinoma progression and survival. Cancer Sci. 2013;104:458-64.

24. Kogo R, Shimamura T, Mimori K, Kawahara K, Imoto S, Sudo T, Tanaka F, Shibata K, Suzuki A, Komune S, et al. Long noncoding RNA HOTAIR regulates polycomb-dependent chromatin modification and is associated with poor prognosis in colorectal cancers. Cancer Res. 2011;71:6320-6.

25. Geng YJ, Xie SL, Li Q, Ma J, Wang GY. Large intervening non-coding RNA HOTAIR is associated with hepatocellular carcinoma progression. J Int Med Res. 2011;39:2119-28.

26. Ishibashi M, Kogo R, Shibata K, Sawada G, Takahashi Y, Kurashige J, Akiyoshi S, Sasaki S, Iwaya T, Sudo T, et al. Clinical significance of the expression of long non-coding RNA HOTAIR in primary hepatocellular carcinoma. Oncol Rep. 2013;29:946-50.

27. Kim K, Jutooru I, Chadalapaka G, Johnson G, Frank J, Burghardt R, Kim S, Safe $S$. HOTAIR is a negative prognostic factor and exhibits pro-oncogenic activity in pancreatic cancer. Oncogene. 2013;32:1616-25.

28. Chen G, Wang J, Cui Q. Could circulating miRNAs contribute to cancer therapy? Trends Mol Med. 2013;19:71-3. 